\title{
An Improved LFS Engine for Physical Layer Security Augmentation in Cognitive Networks
}

\author{
Paul K. Harmer and Michael A. Temple \\ US Air Force Institute of Technology \\ Wright-Patterson AFB, OH 45433 USA \\ Email: [Paul.Harmer*, Michael.Temple]@afit.edu \\ *Correspondence POC
}

\begin{abstract}
Security and privacy within existing wireless architectures remain a major concern and may be further compounded when considering multi-node wireless cognitive networks. However, the same computational capabilities that enable cognitive transceiver operation can also be used to enhance physical-layer security at each node. The approach here uses RF Distinct Native Attribute (RF-DNA) features that embody unique statistical properties of received RF emissions. The baseline system uses a Multiple Discriminant Analysis, Maximum Likelihood (MDA/ML) process to classify devices by exploiting RF-DNA uniqueness that enables serial number discrimination. MDA/ML limitations, to include a lack of feature relevance indication, are addressed using a previously investigated Learning From Signals (LFS) process. Of significance here is the expansion of LFS capability which will be readily implementable in envisioned cognitive network architectures. By coupling Kernel Regression (KR) with a Differential Evolution (DE) genetic algorithm, LFS is able to "learn" an improved model of the signal environment. Results here for experimentally collected 802.11a WiFi signals demonstrate recent improvements to the LFS engine that enable it to operate more effectively within a higher-dimensional RFDNA feature space. The addition of a fractional Euclidean Distance (ED) similarity metric and vector class labeling provide improvement of $9 \%$ to $23 \%$ in average percent correct classification over the earlier LFS implementation.
\end{abstract}

\section{INTRODUCTION}

Security and privacy concerns within wireless networks are well documented and will likely extend to greater effect in ad hoc, adaptive, wireless cognitive networks. For example, vulnerability of Wireless Access Points (WAP) is among the top 10 Information Technology (IT) security concerns [1] and will be compounded when considering the number of wireless nodes (access points) existing within a cognitive network.

There have been many proposed wireless security improvements, with a majority residing in upper layers (three or above) of the Open Systems Interconnect (OSI) network stack [2]. These bit-level protection mechanisms are the focus of many spoofing attacks. Thus, Radio Frequency (RF) air monitoring is proposed here using OSI Physical layer (PHY) information to implement a stand-alone, or augment existing, network security mechanisms. The potential of this approach has been successfully demonstrated for 802.11 [3][5], GSM [6], WiMAX [5], [7], Bluetooth [8], RFID [9], and ZigBee devices [10], [11] using a localized WAP air monitoring. The potential could be even greater in applications using cognitive radios at the network's "edge" to provide native on-board PHY security air monitoring [12]. As PHY security augmentation continues to gain momentum, so does research aimed at integrating mechanisms across PHY and bit-level OSI layers [10] and evaluating robustness of various PHY fingerprinting methods against attack [13].

Although relatively robust and simple to implement, Multiple Discriminant Analysis with Maximum Likelihood (MDA/ML) estimation is limited by 1) the linear algebra formulation, 2) lack of insight into input feature relevance, and 3 ) the static nature of resultant multi-signal system models. To address these limitations and build an adaptive framework that can better capitalize on future cognitive network capabilities, improvement to a previously introduced Learning from Signals (LFS) classification engine is considered here. The LFS framework in [5] is denoted as LFS-V1 and used for baseline comparison with the new LFS-V2 process developed herein.

The proposed LFS-V2 process maintains the Kernel Regression (KR) and Differential Evolution (DE) genetic algorithm learning elements of LFS-V1 and includes specific improvements consisting of: 1) a fractional Euclidean Distance (ED) similarity metric [14], [15] that provides improved performance in higher dimensional spaces, and 2) vector class labeling to mitigate the effect of unequal weighting/cost that occurs with integer labeling. Considering the average percent correct classification $(\% C)$ improvement demonstrated herein, and envisioned improvement for modifications that are underway, the improved LFS engine is a viable alternative for PHY-based cognitive network security. The vision remains focused on improving LFS performance such that MDA/ML discrimination performance is matched, or exceeded, while simultaneously overcoming MDA's limitations.

\section{BACKGROUND}

The PHY air monitoring approach here exploits RF Distinct Native Attribute (RF-DNA) features that embody unique statistical properties of received RF emissions [5], [6]. It has been shown that differences in devices, such as caused by differences in manufacturer, lot number, chip set, etc., induce variation in the transmitted waveform. These differences can be captured in statistical RF-DNA and used to discriminate devices at the serial number level [4] and used for verification, i.e., granting authorized devices network access while rejecting impersonating rogue devices. 
The mathematical heart of LFS is Kernel Regression (KR). Unlike ordinary least squares modeling that produces an overall system model, KR is a non-parametric technique that produces a set of smaller models that are used in collaboration with past example inputs. This is done by comparing past samples $\left(\mathbf{x}_{\mathbf{i}}\right)$ with newly received signals $(\mathbf{q})$ using a distance metric. This distance metric is generally weighted by a kernel shape such as the Gaussian kernel used here. In this way, those samples that are most similar to the received burst RFDNA produce a high contribution, while those the Gaussian tail region are effectively eliminated.

This Gaussian weighting is done on each RF-DNA classification feature input dimension, of which there can be 100 or more. Therefore, each input dimension requires an $h_{i}$ Gaussian bandwidth parameter, related to variance. Finally, the inputs across all the multi-variate Gaussians an averaged using the Nadaraya-Watson Kernel Regression estimator (4) to produce an estimated class label, $\widehat{y}[16]$.

$$
\begin{gathered}
K\left(d_{\mathbf{H}}\left(\mathbf{x}_{\mathbf{i}}, \mathbf{q}\right)\right)=\exp ^{-0.5 \cdot d_{\mathbf{H}}\left(\mathbf{x}_{\mathbf{i}}, \mathbf{q}\right)}, \\
\mathbf{H}=\operatorname{diag}\left(h_{1}, h_{2}, \ldots, h_{N_{F}}\right), h_{i} \geq 0 \forall i \\
d_{\mathbf{H}}\left(\mathbf{x}_{\mathbf{i}}, \mathbf{q}\right)=\left(\mathbf{x}_{\mathbf{i}}-\mathbf{q}\right)^{T} \mathbf{H}^{-1}\left(\mathbf{x}_{\mathbf{i}}-\mathbf{q}\right) . \\
\widehat{y}=\frac{\sum_{i=1}^{N_{S}} K\left(d_{\mathbf{H}}\left(\mathbf{x}_{\mathbf{i}}, \mathbf{q}\right)\right) \cdot y_{i}}{\sum_{i=1}^{N_{S}} K\left(d_{\mathbf{H}}\left(\mathbf{x}_{\mathbf{i}}, \mathbf{q}\right)\right)}, \quad N_{S}=N_{D} \cdot N_{B} .
\end{gathered}
$$

The problem with this approach is that the optimum bandwidth parameter, $h_{i}$, in each $N_{F}$ feature dimension that best separates the device bursts is unknown. While some rules-ofthumb exist for estimating the $\mathbf{H}$, they become less reliable for higher dimensional spaces [14], [15]. Therefore, LFS uses differential evolution (DE) to search for the best $\mathbf{H}$ using the learning power of the genetic algorithm (Fig. 1).

In this non-parametric, supervised learning system, a set of training samples $\mathbf{X}$ and associated class labels $\mathbf{Y}$ are input to the DE-optimized KR learning system. Within this, a population, $\mathbf{P}_{\mathbf{G}}$, of $\mathbf{H}$ values are evolved using DE vector operations. These population members are used to perform KR and the best population member is the single $\mathbf{H}$ that produces the minimum error between known class membership labels $\mathbf{Y}$ and KR-estimated class labels $\hat{\mathbf{Y}}$. After a predefined amount of learning, or an error minimization criterion is met, the process yields a system model consisting of the best learned $\mathbf{H}$ and the past training examples $\mathbf{X}$ and $\mathbf{Y}$. This model is then used within $\mathrm{KR}$ to map a previously unseen sample $\mathbf{Q}$ into label space. It is within this mapped label space that device classification and/or verification can be implemented.

A major benefit resulting from the search for a best $\mathbf{H}$ is that individual $h_{i} \in \mathbf{H}$ values indicate the relative importance

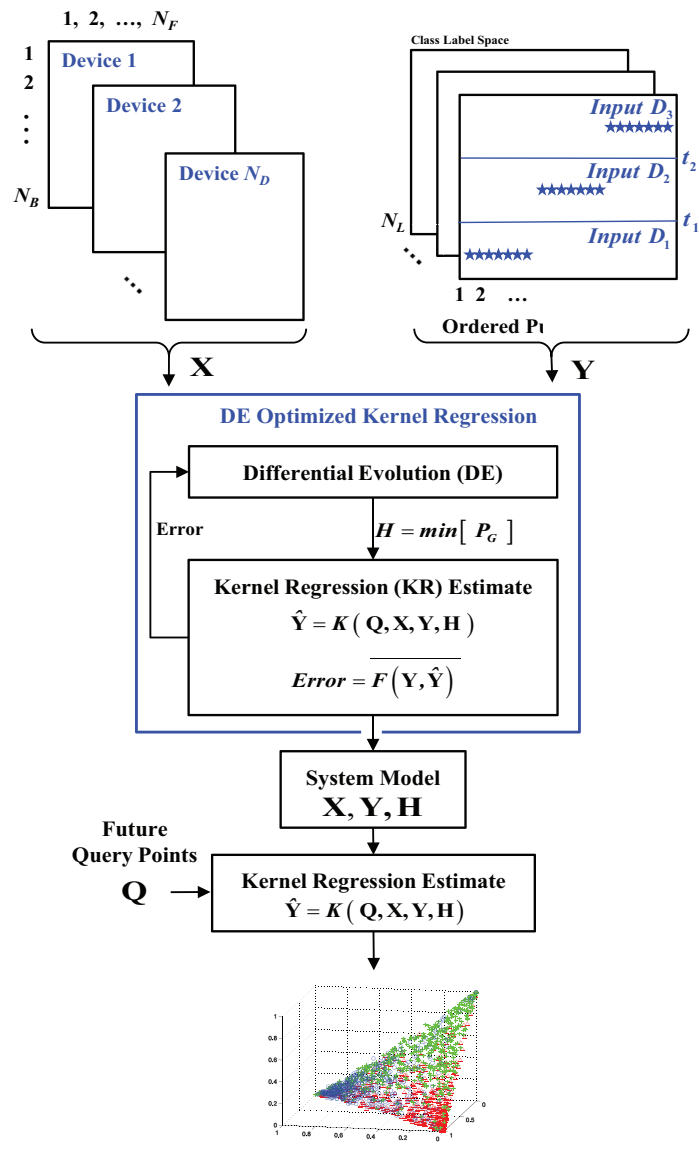

Fig. 1. DE-optimized LFS classification process.

in system performance [14]. A smaller $h_{i}$ value indicates a tight cluster in feature space which implies a better feature for identifying class membership while a larger value indicates greater cluster spread and less significance.

\section{Demonstration Methodology}

To demonstrate LFS system performance, signals for $N_{D}=$ 3 like-model 802.11a Cisco PCMCIA devices were experimentally collected at $S N R \approx 30 \mathrm{~dB}$ and processed as described in [5]. For each device, $N_{B}=500$ bursts were collected for training and an additional $N_{B}=500$ subsequently collected and held-out for testing classification performance. The collected bursts were SNR scaled using Additive White Gaussian Noise (AWGN) to simulate varying wireless cognitive network channel conditions. At each SNR, RF-DNA was generated using time domain (amplitude, phase, and frequency) characteristics to produce $N_{F}=117$ features for each burst. The same resultant $\mathbf{X}_{500 \times 117}$ and $\mathbf{Q}_{500 \times 117}$ RF-DNA feature sets were input to MDA/ML and the various implementations of the LFS engine for comparative performance assessment.

\section{A. Similarity Metric: Fractional Euclidean Distance}

The core of the Kernel Estimation process is a similarity, or distance metric. The most natural distance to compare two real-valued vectors, and the most common metric found 
in the literature, is the Euclidean distance (ED) [14], [16]. However, ED becomes less effective at measuring similarity as the number of features increases due to the curse of dimensionality [17]. As the dimensionality increases, the supervised learning dataset becomes sparser and sparser within the exponentially increasing volume of the feature space. The result is that the distance to similar bursts becomes just as far as to every other burst, rendering an ED similarity metric useless. But, the fractional ED in (5) offers improved performance in high dimensional spaces over the standard ED [17]-[19]. We have adopted this as our improved distance metric for the high dimensional RF-DNA feature spaces.

$$
d_{p, r}(\mathbf{x}, \mathbf{q})=\left(\sum_{i=1}^{N_{F}}\left|x_{i}-q_{i}\right|^{p}\right)^{\frac{1}{r}}
$$

\section{B. Signal Mapping: Vector Class Labels}

The KR system maps input RF bursts from RF-DNA feature space to class label space using a similarity metric scaled by $\mathbf{H}$ and the KR estimator. Results for the LFS-V1 process in [5] were based on integer labels such that all bursts were mapped to a number line. This process functionally works for $N_{C}=2$ and $N_{C}=3$ class problems commonly found in the literature [14] but is ineffective for higher class problems. Additionally, integer labeling creates unequal weighting, or an implied cost function, as a " 3 " label is three times larger than a " 1 " label in the averaging effect of (4). This can have an especially adverse effect when relatively larger labeled outlier bursts appear similar to in-class bursts. So, system performance was improved using unit vector labels:

$$
\begin{aligned}
& \mathbf{Y}=\mathbb{F}^{N_{C}} \\
& y_{1}=e_{1}=\{1,0,0, \ldots, 0\} \\
& y_{2}=e_{2}=\{0,1,0, \ldots, 0\} \\
& \vdots \\
& y_{N_{C}}=e_{N_{C}}=\{0,0,0, \ldots, 1\}
\end{aligned}
$$

Device bursts with vector labeling are mapped from feature space to an $N_{C}-1$ hyper-polygon in $\mathbb{R}^{N_{C}}$ space. This is illustrated in Fig. 2 for an a $N_{C}=3$-class problem which maps to a 2-D triangle in 3-D space.

Unit vector labeling provides multiple advantages, including: 1) class labels are equally weighted and equal distance apart which eliminates an implied cost function, and 2) the resultant vector mapping provides a convenient space for visualization, classification, and verification operations. The resultant estimated system response, or class mapping, is of the form $[x, y, z]$ and provides a measure of how much a given burst "looks like" a member of each class. For example, $[x=0.7, y=0.1, z=0.2]$ reflects that the input looks most like Class 1 (70\%) and least like Class 2 (10\%).

\section{Probability-Based Classification}

The LFS-V1 system with integer labeling and number line mapping enabled classification (device discrimination) using a simple distance metric and thresholding process. Extending the system to vector labeling in LFS-V2, as described in Sect.III-B, allowed extension to multi-dimensional distancebased classification (i.e. a mapped burst's distance to the nearest class label vertex indicated its estimated class membership). In general, a probability-based classifier should perform better than a distance-based classifier by taking into account, not just the projected point location, but also the relative class densities at the projected point. Therefore, to improve LFS system performance further a maximum likelihood classification process was next considered.

Mapping to vector label space using the LFS-V2 process allows for classification using Maximum A-Posteriori (MAP) criteria. This is illustrated using Fig. 2 which shows supervised learning data mapped to the label space using KR and the best learned $\mathbf{H}$ values. In general, the mapped points do not conform to any closed-form standard distribution. Therefore, an estimated PDF (ePDF) is created for each class of mapped data using a parzen window, or Kernel Density Estimation (KDE). KDE utilizes the same Gaussian kernel function for smoothing as used for KR in (1), but it is applied slightly differently to achieve a density estimate [20]. The effect is to create smoothed, bin-less, multi-dimensional histograms for each set of mapped class points within the label space. In the case of a 3-class problem, the KDE result is a 3-D probability overlaid on top of the 2-D mapped input data as shown in Fig. 3 using KR-mapped input bursts in Fig. 2.

A MAP classifier can then be created by finding the class with the maximum ePDF at each point in the label space. This yields classification decision regions and boundaries as shown in Fig. 4 where equal costs and equal prior probabilities are assumed for all classes. Future unseen input burst are then mapped to this space using KR and an associated class label assigned based on MAP assignment.

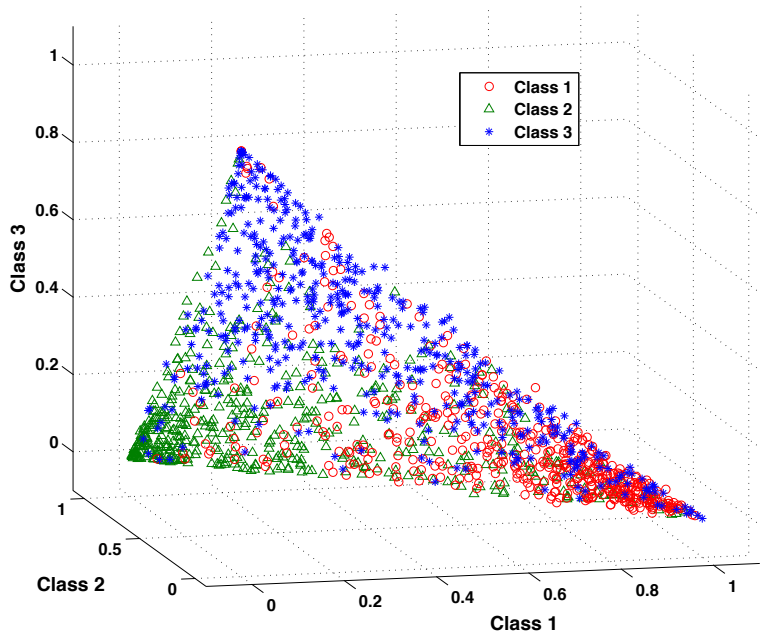

Fig. 2. Representative $N_{C}=3$-class LFS classification space for $802.11 \mathrm{a}$ data at $S N R=12 \mathrm{~dB}$. Each RF-DNA burst is mapped to an $N_{C}-1$ polygon in $\Re^{N_{C}}$ space based on unit vector class labels. 


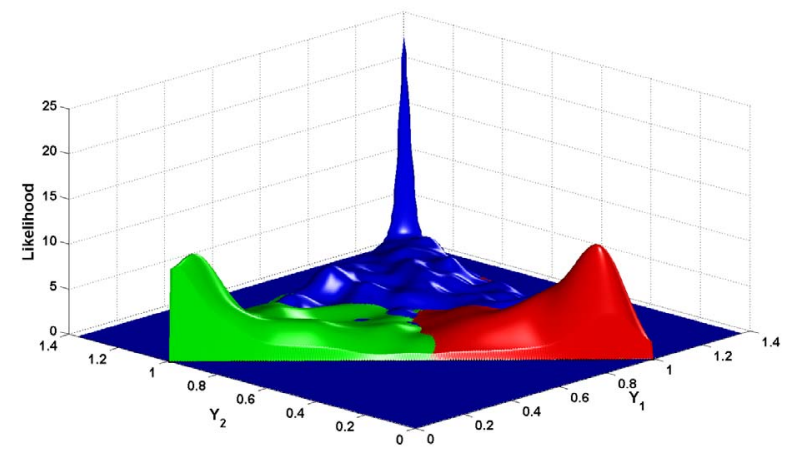

Fig. 3. Representative LFS-V2 (MAP) classification space created using Kernel Density Estimation (KDE) of the KR-mapped input bursts in Fig. 2.

\section{REsults}

Four different classification systems were applied to the same SNR-dependent RF-DNA feature sets. The functional differences in these approaches are summarized in Table I. The results using the improvements in LFS processing between versions compared to the MDA/ML classifier baseline are discussed below.

\section{A. Fractional Euclidean Distance}

The high dimensional $N_{F}=117$ RF-DNA feature space requires use of a similarity metric that is not hampered by the sparseness of this space. The fractional Euclidean distance was selected, but this leaves the requirement to find the best ratio, $p / r$, to use for this approach. For the results in Fig. 5, LFS was used to learn a best $\mathbf{H}$ at $S N R=12 \mathrm{~dB}$ and $S N R=24 \mathrm{~dB}$ and both a similarity metric distance ratio of $p / r=1$ for the "Fractional Euclidean Model" and a $p / r=2$ for the "Euclidean Model." The testing data was then mapped and

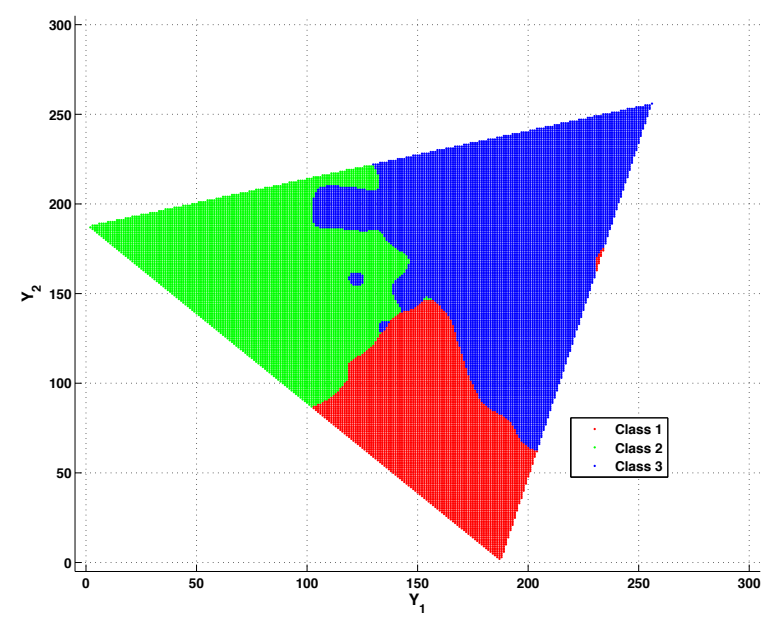

Fig. 4. Resultant MAP classification decision regions and boundary for 802.11a at $S N R=12 \mathrm{~dB}$ data and LFS-V2 (MAP) classification space in Fig. 3.
TABLE I

CHARACTERISTICS OF THE FOUR CLASSIFICATION SYSTEMS USED FOR COMPARATIVE ASSESSMENT.

\begin{tabular}{|c|c|c|c|c|c|}
\hline & \multicolumn{3}{|c|}{ Similarity Metric } & \multicolumn{2}{c|}{ Classifier } \\
\hline & MDA & $(\mathbf{x}-\mathbf{q})^{2}$ & $(\mathbf{x}-\mathbf{q})^{p / r}$ & Distance & ML \\
\hline MDA/ML & $\times$ & & & & $\times$ \\
\hline LFS-V1 & & $\times$ & & $\times$ & \\
\hline LFS-V2 (DM) & & & $\times$ & $\times$ & \\
\hline LFS-V2 (MAP) & & & $\times$ & & $\times$ \\
\hline
\end{tabular}

classified using these $\mathbf{H}$ and a fractional Euclidean distance similarity metric $p / r \in[0.1,2.0]$ in 0.1 steps.

Results indicate that indeed the standard Euclidean distance $(p / r=2)$ produced worst case results for $S N R=24 \mathrm{~dB}$ and are on par with the worst results for $S N R=12 \mathrm{~dB}$. The best results with this $802.11 \mathrm{a}$ dataset were achieved at $p / r=1.1$ for $S N R=12 \mathrm{~dB}$ and $p / r=0.8$ for $S N R=24 \mathrm{~dB}$. Although, at $S N R=24 \mathrm{~dB}$ performance was relatively flat at about $96 \%$ correct over the range $p / r \in[0.7,1.3]$. It can be seen that performance plateaus at higher SNR, where noise was not as much a factor in dispersing the data. At $S N R=12 \mathrm{~dB}$ a more pronounced difference in performance was noted. Even though the best performance was for $p / r=1.1$, results on either side of this point were similar. So, based on a minor performance degradation, but a computational benefit of not having to compute a fractional exponent (a 6x speedup), an exponent ratio $p / r=1$ was selected for the system. This corresponds to a "city block" distance metric, or simply the sum of differences in each dimension between compared bursts.

\section{B. Device Classification Performance}

Average percent correct classification $(\% C)$ was calculated for all systems across a range of $S N R$. At lower $S N R$, all systems expectedly approach $\% C=33 \%$ (random-guess) while for more signal-dominated cases $(S N R \approx 30.0 \mathrm{~dB})$ all systems approach $\% C=100 \%$. At these extremes performance

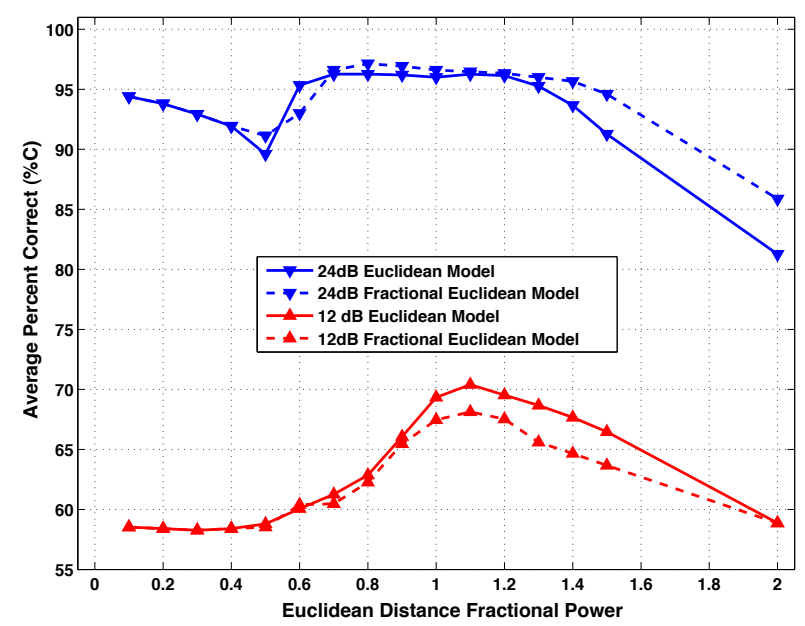

Fig. 5. Classification performance of LFS-V2 with various fractional Euclidean distance $p / r$ exponent values for $S N R=12 \mathrm{~dB}$ and $S N R=24 \mathrm{~dB}$ 802.11a data. 
differences are less dramatic and therefore $S N R \in[6,24] \mathrm{dB}$ was used for comparison. Overall $\% C$ improvement relative to LFS-V1 is shown in Table II for MDA/ML and each LFS-V2 configuration.

As indicated in Table II, the addition of vector labels and the fractional Euclidean distance metric in LFS-V2 (DM) yielded improvements of $9.72 \%$ to $23.30 \%$ in the testing $\% C$ across the range of $S N R$ considered. It was originally hypothesized that addition of a probability-based MAP classifier would further improve system performance. Table II results show this was not the case, with LFS-V2 (MAP) performance at all $S N R$ s, except $S N R=12.0 \mathrm{~dB}$, being slightly poorer than LFS-V2 (DM) using a simple distance-based classifier. At $S N R=12.0 \mathrm{~dB}$, the MAP classifier had an almost $\% \mathrm{C}=2 \%$ increase over the distance approach. The generality of a vector distance approach appears to be a good model across $S N R$ for classification. However, distance-based performance may degrade as the label space dimensionality increases (larger number of classes) and work continues in this area.

\section{CONCLUSION}

Security and privacy within multi-node wireless cognitive networks are a concern and improvement can be made using physical-layer mechanisms. The work here continues development of RF fingerprinting methods and progress has been made in extending applicability of a Learning From Signals (LFS) engine for deployment within a wireless cognitive network. The improved LFS-V2 process maintains Kernel Regression (KR) and Differential Evolution (DE) benefits of the original LFS-V1 process and provides additional improvement with the addition a fractional Euclidean Distance (ED) similarity metric and vector class labeling. Demonstrations here using RF fingerprints from experimentally collected 802.11a WiFi signals show that these additions collectively provide $9 \%$ to $23 \%$ improvement in overall device discrimination performance for $S N R \in[6,24] \mathrm{dB}$.

The vision remains focused on additional LFS improvements such that MDA/ML discrimination performance is matched, or exceeded, while simultaneously overcoming MDA's limitations. LFS model development is currently based on learning a single, system-wide $\mathbf{H}$ that best separates input classes. Analysis suggests that this approach is potentially compromising in that subtle peculiarities within specific class data may be averaged out during $\mathbf{H}$ development. As a next step, provisions are being made to assess performance using class-dependent $\mathbf{H}^{\mathbf{C}}$ vectors. It is hypothesized that this may better separate the classes and better cluster the within-class

TABLE II

ClASSIFICATION PERFORM ANCE Improvement RELATIVE TO LFS-V1: MDA/ML, LFS-V2 (DISTANCE METRIC) AND LFS-V2 (MAXIMUM A Posteriori) CLASSIFIERS.

\begin{tabular}{|c|c|c|c|c|}
\hline SNR (dB) & 6.0 & 12.0 & 18.0 & 24.0 \\
\hline MDA/ML & 9.72 & 14.50 & 18.23 & 20.74 \\
\hline LFS-V2 (DM) & 8.73 & 14.60 & 15.70 & 23.30 \\
\hline LFS-V2 (MAP) & 8.40 & 16.53 & 13.50 & 22.59 \\
\hline
\end{tabular}

bursts with an associated classification performance increase. Additionally, while demonstrated here using experimentally collected 802.11a signals, LFS is a signal agnostic learning system and work continues to applying LFS to other communication signals such as ZigBee, WiMAX, and LTE.

\section{ACKNOWLEDGMENT}

Work sponsored by the Sensors Directorate, Air Force Research Laboratory, Wright-Patterson AFB, OH, and the Laboratory for Telecommunications Sciences, US Department of Defense.

"The views expressed in this article are those of the author(s) and do not reflect official policy of the United States Air Force, Department of Defense or the U.S. Government"

\section{REFERENCES}

[1] Collins, H., "Top 10 Network Security Threats," Government Technology, Sep 2010.

[2] Tarman, T.D. and E.L. Witzke, "Intrusion Detection Considerations for Switched Networks," in Enabling Technologies for Law Enforcement and Security, vol. 4232, no. 1. SPIE, 2001, pp. 85-92.

[3] Brik, V., et al., "Wireless Device Identification with Radio-metric Signatures," in 14th ACM Int'l Conf on Mobile Computing and Networking (MOBICOM08), Sep 2008

[4] Klein, R.W., et al., "Application of Wavelet-Based RF Fingerprinting to Enhance Wireless Network Security," Jour of Communications and Networks, vol. 11, no. 6, pp. 544-555, Dec 2009.

[5] Harmer, P.K., et al., "4G Security Using Physical Layer RF-DNA with DE-Optimized LFS Classification," Journal of Communications Networks, vol. 6, no. 9, pp. 671-681, 2011.

[6] Reising, D.R., et al., "Improved Wireless Security for GMSK-Based Devices Using RF Fingerprinting," Int. J. Electronic Security and Digital Forensics, vol. 3, no. 1, pp. 41-59, Mar 2010.

[7] Williams, M.D., et al., "RF-DNA Fingerprinting for Airport WiMAX Communications Security," in 4th Int'l Conf on Network and Systems Security (NSS10), Sep 2010.

[8] Hall J., et al., "Detecting Rogue Devices in Bluetooth Networks Using Radio Frequency Fingerprinting," in Communications and Computer Networks, 2006, pp. 108-113.

[9] Danev, B., et al., "Physical-Layer ID of RFID Devices," in 18th USENIX Security Sym, Berkeley, CA, USA, Sep 2009, pp. 199-214.

[10] Ramsey, B.W., et al., "PHY Foundation for Multi-Factor ZigBee Node Authentication," in 2012 Global Communications Conf (GLOBECOM12), Dec 2012.

[11] Dubendorfer, C.K., et al., "An RF-DNA Verification Process for ZigBee Networks," in 2012 Military Comm Conf (MILCOM12), Oct 2012.

[12] Moore, M.R. and M.A. Buckner, "Learning-From-Signals on Edge Devices," Instrumentation Measurement Magazine, IEEE, vol. 15, no. 2, pp. 40-44, April 2012.

[13] Danev, B., et al., "Attacks on Physical-Layer Identification," in 3rd ACM Conf on Wireless Network Security (WISEC10. ACM, 2010.

[14] Hand, D.J., Kernel Discriminant Analysis. John Wiley \& Sons, 1982.

[15] Kwon, H. and P. Gurram, "Optimal Kernel Bandwidth Est for Hyperspectral Kernel-Based Anomaly Det," in 2010 IEEE Int'l Geoscience and Remote Sensing Sym (IGARSS10), Jul 2010, pp. 2812 -2815.

[16] Goutte, C. and J. Larsen, "Adaptive Metric Kernel Regression," vol. 26, no. 1/2, pp. 155-167, 2000.

[17] Deza, M.M. and E. Deza, Encyclopedia of Distances. BerlinHeidelberg: Springer-Verlag, 2009.

[18] Aggarwal, C.C., et al., "On the Surprising Behavior of Distance Metrics in High Dimensional Space," in 8th Int'l Conference on Database Theory (ICDT01), Jan 2001, pp. 420-434.

[19] Jimenez, L.O. and D.A. Landgrebe, "Supervised Classification in HighDimensional Space: Geometrical, Statistical, and Asymptotical Properties of Multivariate Data," IEEE Trans on Systems, Man, and Cybernetics, Part C: Apps and Reviews, vol. 28, no. 1, pp. 39-54, Feb 1998.

[20] Duda, R.O., et al., Pattern Classification, 2nd ed. Wiley, Nov 2001. 